\title{
Dificultades en el neurodesarrollo con repercusión en el ámbito educativo en menores con cardiopatías congénitas: revisión sistemática
}

\author{
Difficulties in neurodevelopment with repercussions in the educational \\ environment in minors with congenital cardiopathies: systematic review
}

\begin{abstract}
Resumen
La supervivencia de los recién nacidos con cardiopatías congénitas ha aumentado en las últimas décadas. Tras garantizar esto, estudios científicos empezaron a evidenciar que estos menores presentaban lentificaciones en algunas áreas del neurodesarrollo, haciéndose más palpables en edades posteriores. Se realiza una revisión sistemática para conocer en profundidad los estudios existentes sobre el neurodesarrollo de menores con cardiopatías congénitas, sin anomalías cromosómicas asociadas y sometidos a intervención quirúrgica, consultándose 149 investigaciones ubicadas en las bases de datos de Scopus y Pubmed desde 2008 hasta 2018. Se analizaron en profundidad quince estudios que cumplían con los criterios de elegibilidad. Los estudios científicos muestran que las personas con cardiopatías congénitas obtienen puntuaciones inferiores a la media en algunas áreas del neurodesarrollo (lenguaje y motor). Es necesario que los menores con cardiopatías congénitas se deriven a los servicios de atención temprana para prevenir las dificultades que puedan generarse en el ámbito escolar.
\end{abstract}

Palabras clave

Cardiopatías congénitas, neurodesarrollo, intervención quirúrgica, atención temprana, adolescentes, niños.

\begin{abstract}
The survival of newborns with congenital heart disease has increased in recent decades. After guaranteeing this, scientific studies began to show that these children presented slowness in some areas of neurodevelopmental, becoming more palpable at later ages. A systematic review is carried out to gain in-depth knowledge of the existing studies on the neurodevelopmental of children with congenital heart disease, without associated chromosomal anomalies and underwent surgery, consulting 149 studies located in the databases of Scopus and Pubmed from 2008 to 2018. Fifteen studies that met the eligibility criteria were analyzed in depth. Scientific studies show that people with congenital heart disease obtain lower than average scores in some areas of neurodevelopmental (language and motor). Children with congenital heart disease need to be referred to early care services to prevent difficulties that may arise in the school setting.
\end{abstract}

\section{Keywords}

Congenital heart disease, neurodevelopmental, surgical intervention, early care, teenagers, children.

\author{
$M^{a}$ del Rosario Mendoza \\ Carretero \\ <mamendoz@ucm.es>
}

Universidad Complutense de Madrid. España

\section{Susana Ares Segura}

<susana.ares@salud.madrid.org>

Hospital Universitario La Paz. España

\section{Belén Sáenz-Rico de \\ Santiago}

<bsaenzri@edu.ucm.es>

Universidad Complutense de Madrid. España

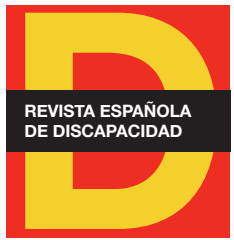

Para citar:

Mendoza, M. R. et al. (2019): "Dificultades en el neurodesarrollo con repercusión en el ámbito educativo en menores con cardiopatías congénitas: revisión sistemática". Revista Española de Discapacidad, 7 (II): 43-53.

Doi: <https://doi.org/10.5569/23405104.07.02.03>

Fecha de recepción: 12-02-2018 Fecha de aceptación: 30-10-2019 


\section{Introducción}

La American Heart Asociation (AHA, 2018) informa de que en Estados Unidos cerca de 40.000 niños nacen con cardiopatías congénitas, con una tasa de incidencia entre 8 y 10 por cada 1000 nacidos vivos.

Estos defectos cardiacos pueden requerir intervención quirúrgica durante el primer año de vida (Greco, 2016), conduciendo a frecuentes y largas estancias hospitalarias (Pasquali et al., 2011) y presentan una mayor tasa de mortalidad (Robbins et al., 2007), contando con entre el $5 \%$ y el $10 \%$ de las muertes neonatales (Ferencz, C. y Neil, C. A., 1992).

Algunas de estas anomalías cardiacas son benignas, siendo consideradas como cardiopatías congénitas menores, pues se pueden cerrar por sí solas, sin necesidad de intervención quirúrgica, como puede suceder con el foramen oval, ubicado en la comunicación interauricular (CIA) (Cardiopediamarañón, 2017).

En las últimas décadas los avances existentes en las técnicas quirúrgicas y los cuidados perioperatorios han propiciado un aumento en la tasas de supervivencia de los recién nacidos con cardiopatías (Williams et al., 2015) aunque se reconocen morbilidades en el neurodesarrollo (Martínez-Biarge et al., 2013) que posiblemente sean derivadas de la fisiología, la anestesia, la duración de la cirugía por circulación extracorpórea (CEC), la prolongación de la estancia hospitalaria, etc. (Marino et al., 2012; Gaynor et al., 2007). Por este motivo, autores como Martínez-Biarge et al. (2013) indican que la etiología de las alteraciones en el desarrollo neurológico puede ser multifactorial.

La AHA resalta que la prevalencia y la gravedad de estas morbilidades aumenta con severidad en los niños con CC (García et al., 2018) intervenidos quirúrgicamente mediante CEC. La literatura actual señala que los lactantes con CC corren mayor riesgo de obtener peores resultados en el desarrollo neurológico tanto en el dominio cognitivo como en el motor, tanto en la infancia temprana como en la tardía (Harvey et al., 2013).

Por lo tanto, el objetivo de este estudio es realizar una revisión de la literatura de las investigaciones publicadas en la última década (2008-2018) para sistematizar la información sobre el neurodesarrollo de los menores con CC, sin síndromes cromosómicos relacionados, sometidos a intervención quirúrgica en la primera infancia, a fin de proporcionar una visión general centrada en las morbilidades y comorbilidades que pueden desencadenarse en el ámbito escolar por la propia cardiopatía.

\section{Metodología}

Se realizó una búsqueda bibliográfica en Scopus y PubMed de todas las investigaciones publicadas sobre esta temática desde 2008 hasta noviembre de 2018. Se utilizaron términos de búsqueda tanto en castellano ("desarrollo motor", "desarrollo del lenguaje", "desarrollo cognitivo", "neurodesarrollo", "psicomotricidad", "niños", "CEC") como en inglés ("neurodevelopmental", "early care", "congenital heart disease", "learning", 
"cognitive", "language", "development", “CEC", "motor", "children”, "preschoolers " "comorbidity", "morbility,", "neurocognitive") que permitieron identificar los artículos de interés en distintas revistas (The Journal of Pediatrics, The Journal of Thoracic and Cardiovascular Surgery, Progress in Pediatric Cardiology, Pediatric Cardiac, Circulation, Seminars in Fetal \& Neonatal Medicine, Developmental Medicine \& Child Neurology...).

Se incluyeron los estudios si: a) la muestra presentaba CC, b) todos habían sido sometidos a intervención quirúrgica en la primera infancia (bypass u otras técnicas de cirugía cardiaca), c) analizaban el neurodesarrollo de aquellos que presentaban CC, d) las investigaciones habían sido publicadas entre 2008-2018.

Se excluyeron los artículos si: a) carecían de muestra con CC, b) la muestra con CC presentaba anomalías cromosómicas o genéticas y/o discapacidad, c) contaban con muestra prematura (< 36 semanas de edad gestacional) y d) no habían sido sometidos a intervención quirúrgica en los primeros años de vida.

Se consultaron 143 estudios y se incluyeron 15, los cuales cumplían con los criterios de elegibilidad. A continuación, en el cuadro 1 , se muestran las investigaciones evaluadas hasta el momento a través de un diagrama de flujo de la información que cuenta con las fases de identificación, de cribado, de elegibilidad y de inclusión, propias de la revisión sistemática.

Se identificaron 143 registros en las búsquedas realizadas y 5 citas adicionales pertenecientes al $24^{\text {th }}$ World Congress on Ultrasound in Obstetrics and Gynecology, al Simposium Cardiovascular Medicine y tres a capítulos ubicados en Congenital Heart Disease. De todos los estudios consultados hasta el momento se ha procedido a la anulación de 9 de ellos por estar duplicados, quedando 139 registros cribados. De esos se eliminaron 22 porque no cumplían con los criterios de inclusión.

Se analizaron en profundidad 117 artículos para su elegibilidad, eliminándose 102 artículos a texto completo porque no cumplían con los parámetros establecidos (muestra, edad, intervención quirúrgica...). Por último, destacar que esta revisión ha garantizado la inclusión de 15 artículos, que fueron analizados en profundidad.

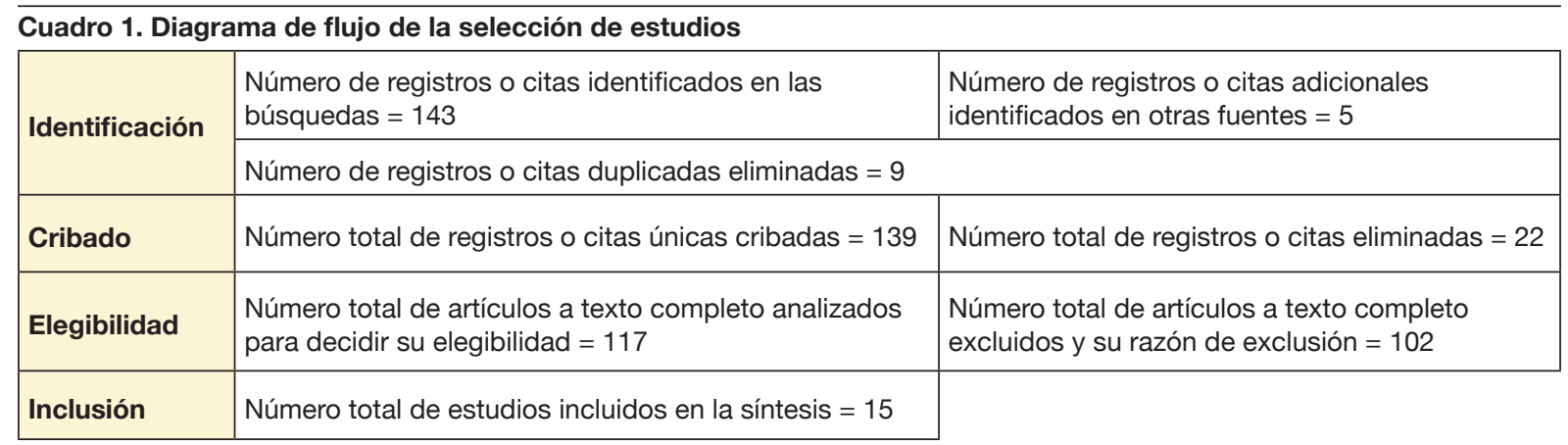

Fuente: elaboración propia.

Los 15 estudios científicos que cumplían con los criterios de inclusión se analizaron individualmente. Las características de estos se muestran en la tabla 1. 


\begin{tabular}{|c|c|c|c|c|}
\hline $\begin{array}{l}\text { Autor y } \\
\text { año de } \\
\text { publicación }\end{array}$ & País & $\begin{array}{c}\text { Características de la muestra, } \\
\text { edad (meses), sexo (masculino/ } \\
\text { femenino) }\end{array}$ & $\begin{array}{l}\text { Herramientas } \\
\text { de recogida de } \\
\text { datos }\end{array}$ & Resultados \\
\hline $\begin{array}{l}\text { Polat et al., } \\
\quad 2011\end{array}$ & Turquía & $\begin{array}{l}127 \mathrm{~N} \text { total } \\
76 \mathrm{CC} \\
46 \mathrm{HN}(24 / 22) \\
30 \mathrm{HI}(19 / 11) \\
51 \mathrm{GC}(22 / 29) \\
\text { Edad: } 1-72 \\
\end{array}$ & TD II & $\begin{array}{l}\text { HI presentó más fallos en el área motora gruesa } \\
\text { y fina que el HN y GC. Asimismo, mostró un } \\
\text { desarrollo más lento en el área del lenguaje que } \\
\text { GC. }\end{array}$ \\
\hline $\begin{array}{l}\text { Sananes et } \\
\text { al., } 2012\end{array}$ & Canadá & $\begin{array}{l}106 \mathrm{~N} \text { total } \\
\text { Edad: } 8-24 .\end{array}$ & $\begin{array}{l}\text { BSID-II MDI } \\
\text { PDMS } \\
\text { ASQ }\end{array}$ & $\begin{array}{l}\text { PDMS Edad: } 8(M F=91 \pm 13 ; M G=78 \pm 16) ; 24 \\
(M F=84 \pm 16 ; M G=91 \pm 13)\end{array}$ \\
\hline $\begin{array}{l}\text { Schaefer et } \\
\text { al., } 2013\end{array}$ & $\begin{array}{l}\text { Reino } \\
\text { Unido }\end{array}$ & $\begin{array}{c}99 \mathrm{~N} \text { total } \\
59 \text { CC }(29 \mathrm{CCA}, 30 \mathrm{CCC}) \\
40 \mathrm{GC} \\
\text { Edad: } 156-192(25 / 34)\end{array}$ & $\begin{array}{c}\text { WISC-IV } \\
\text { ROCFT } \\
\text { ZNA } \\
\text { HRQoL } \\
\text { Prueba Beery- } \\
\text { Buktenica del } \\
\text { desarrollo de } \\
\text { la integración } \\
\text { visomotriz. } \\
\end{array}$ & $\begin{array}{l}16 \% \text { CC muestra dificultades en la lecto- } \\
\text { escritura y en las matemáticas; } 20 \% \text { CC } \\
\text { necesita apoyo extra; } 88 \% \text { CC acude a la } \\
\text { escuela con regularidad. }\end{array}$ \\
\hline $\begin{array}{l}\text { Chen et al., } \\
2015 .\end{array}$ & EE.UU & $\begin{array}{l}24 \mathrm{~N} \text { total } \\
10 \mathrm{CC}(7 / 3) \\
14 \mathrm{GC}(4 / 10) \\
\text { Edad: } 3\end{array}$ & BSID II & $\begin{array}{l}\text { GC área cognitiva: } 99,29 \pm 10,54 \text { y } 95,86 \pm \\
10,26 \text { en el dominio motor. } \\
\text { CC área cognitiva: } 88.50 \pm 18.11 \text { y } 90.10 \pm 7.36 \\
\text { en el dominio motor. }\end{array}$ \\
\hline $\begin{array}{l}\text { Hallioglu et } \\
\text { al., } 2015\end{array}$ & Turquía & $\begin{array}{l}61 \mathrm{~N} \text { total }(30 / 31) \\
37 \mathrm{CC} \\
\text { Edad: } 1-41(17 / 20) \\
24 \mathrm{GC} \\
\text { Edad: } 1-41(13 / 11) \\
\end{array}$ & BSID III & $\begin{array}{l}\text { CC: } 87,36 \pm 16,79 \text { cognitivo; } 87,63 \pm 14.15 \\
\text { lenguaje; } 86,66 \pm 17,95 \text { motor. GC: } 101,18 \pm 11,88 \\
\text { cognitivo; } 104,95 \pm 13,65 \text { lenguaje; } 102,92 \\
\pm 12,54 \text { motor. }\end{array}$ \\
\hline $\begin{array}{l}\text { Murphy et al., } \\
2015\end{array}$ & EE.UU & $\begin{array}{c}36 \mathrm{~N} \text { total } \\
18 \mathrm{CC}(7 / 11)(12 \text { TOF y } 6 \text { D-TGA }) \\
18 \text { sin CC }(8 / 10) \\
\text { Edad: } 132-264\end{array}$ & $\begin{array}{l}\text { WISC-IV } \\
\text { WAIS-IV } \\
\text { CBCL } \\
\text { YSR } \\
\text { ASR }\end{array}$ & $\begin{array}{l}\text { Las personas con CC presentaron una } \\
\text { velocidad de procesamiento a gran escala, un } \\
\text { razonamiento perceptivo inferior al GC. }\end{array}$ \\
\hline $\begin{array}{l}\text { Sarrechia et } \\
\text { al., } 2015\end{array}$ & Bélgica & $\begin{array}{l}63 \mathrm{~N} \text { total }(32 / 21) \\
17 \mathrm{UVH} \\
\text { Edad: } 108(13 / 4) \\
46 \mathrm{BiVH} \\
\text { Edad: } 108(19 / 17) \\
\end{array}$ & $\begin{array}{l}\text { WISC-III } \\
\text { NePsy. } \\
\text { CBCL }\end{array}$ & $\begin{array}{l}\text { UVH muestran diferencias significativas en las } \\
\text { funciones ejecutivas y puntuaciones inferiores } \\
\text { en la memorización. }\end{array}$ \\
\hline $\begin{array}{l}\text { Goldsworthy } \\
\text { et al., } 2016\end{array}$ & EE.UU & $\begin{array}{c}105 \mathrm{~N} \text { total } \\
\text { Edad: } 24,2 \pm 1,4(63 / 42)\end{array}$ & $\begin{array}{l}\text { BSID III } \\
\text { ITSEA }\end{array}$ & $\begin{array}{l}\text { Se obtienen peores puntuaciones en el } \\
\text { neurodesarrollo de los menores con CC. }\end{array}$ \\
\hline $\begin{array}{l}\text { Medoff- } \\
\text { Cooper et al., } \\
2016\end{array}$ & EE.UU & $\begin{array}{c}72 \mathrm{~N} \text { total } \\
\text { Edad: } 6-12(50 / 22)\end{array}$ & BSID III & $\begin{array}{l}\text { MDI está más afectada que PDI. } \\
\text { A los } 6 \text { meses: } \bar{X} 92 \pm 10 \mathrm{MDI} ; \bar{X} 81 \pm 14 \text { PDI. } \\
\text { A los } 12 \text { meses: } \bar{X} 94 \pm 12 \mathrm{MDI} ; \bar{X} 80 \pm 16 \text {. }\end{array}$ \\
\hline $\begin{array}{l}\text { Gerstle et al., } \\
\quad 2016\end{array}$ & EE.UU & $\begin{array}{c}143 \mathrm{~N} \text { total } \\
\text { Edad: } 96-192(86 / 57)\end{array}$ & $\begin{array}{l}\text { BRIEF } \\
\text { CBCL } \\
\text { Peds-QL } \\
\text { WISC-IV } \\
\text { QOL }\end{array}$ & $\begin{array}{l}\text { CIC obtiene menores puntuaciones en } \\
\text { la regulación de conducta. VU alcanzan } \\
\text { puntuaciones inferiores en competencia escolar } \\
(\bar{X}=39,9) \text { en comparación con aquellos que } \\
\text { tienen lesiones en los } 2 \text { ventrículos }(\bar{X}=43,7) \text {. }\end{array}$ \\
\hline
\end{tabular}




\begin{tabular}{|c|c|c|c|c|}
\hline $\begin{array}{l}\text { Autor y } \\
\text { año de } \\
\text { publicación }\end{array}$ & País & $\begin{array}{l}\text { Características de la muestra, } \\
\text { edad (meses), sexo (masculino/ } \\
\text { femenino) }\end{array}$ & $\begin{array}{l}\text { Herramientas } \\
\text { de recogida de } \\
\text { datos }\end{array}$ & Resultados \\
\hline $\begin{array}{l}\text { Latal et al., } \\
\quad 2016\end{array}$ & Suiza & $\begin{array}{c}50 \mathrm{~N} \text { total } \\
\text { Edad: } 12-48(33 / 17)\end{array}$ & $\begin{array}{l}\text { Monitor cerebral } \\
\text { BRM2 } \\
\text { BSID II } \\
\text { WISC IV } \\
\text { M-ABC-2 }\end{array}$ & $\begin{array}{l}12 \text { meses: } \bar{X} \text { 92,0 cognitiva; } \bar{X} 75,1 \text { psicomotor. } \\
48 \text { meses: el área cognitiva no difería del grupo } \\
\text { normativo aunque el psicomotor es inferior al } \\
\text { esperado por edad. }\end{array}$ \\
\hline $\begin{array}{l}\text { Sterken et al., } \\
\quad 2016\end{array}$ & Bélgica & $\begin{array}{l}184 \mathrm{~N} \text { total }(107 \mathrm{CC}, 77 \mathrm{GC}) \\
\text { Edad: } 48 \text { a } 84(100 / 84)\end{array}$ & $\begin{array}{l}\text { WPPSI-R } \\
\text { CBCL } \\
\text { Prueba Beery- } \\
\text { Butenica del } \\
\text { desarrollo de } \\
\text { la integración } \\
\text { visomotriz. }\end{array}$ & $\begin{array}{l}\text { No existen diferencias entre la } 1^{\mathrm{a}} \text { ( } 4 \text { años) y la } 2^{\mathrm{a}} \\
\text { (7 años) valoración del grupo con CC: Cl }(94,6 \text {; } \\
95,6) \text {, verbal }(95,6 ; 97,5) \text {. }\end{array}$ \\
\hline $\begin{array}{l}\text { Rollins et al., } \\
\quad 2017\end{array}$ & EE.UU & $\begin{array}{c}61 \mathrm{~N} \text { total } \\
48 \mathrm{CC} \\
13 \mathrm{GC} \\
\text { Edad: } 12(28 / 20)\end{array}$ & $\begin{array}{c}\text { BSID-II } \\
\text { Inventarios } \\
\text { de Desarrollo } \\
\text { Comunicativo de } \\
\text { Mac Arthur-Bates }\end{array}$ & $\begin{array}{l}\text { EI PDI y el MDI fueron más bajos en el grupo } \\
\text { con CC }(85,8 \pm 16,3 \mathrm{PDI} ; 94,7 \pm 11,3 \mathrm{MDI}) \text { que } \\
\text { en GC }(96,2 \pm 9,8 \mathrm{PDI} ; 101,8 \pm 11,7 \mathrm{MDI})\end{array}$ \\
\hline $\begin{array}{l}\text { González- } \\
\text { González et } \\
\text { al., } 2018\end{array}$ & México & $\begin{array}{c}20 \mathrm{~N} \text { total }(11 / 9) 12 \text { adolescentes, } \\
8<13 \text { años. } \\
9 \mathrm{CCs} \\
11 \mathrm{CCc} \\
\text { Edad:84-192 } \\
\end{array}$ & $\begin{array}{l}\text { Neuropsi } \\
\text { Test de } \\
\text { diferencias de } \\
\text { proporciones }\end{array}$ & $\begin{array}{l}\text { Existen diferencias entre CCs y CCc en } \\
\text { atención, funciones ejecutivas, memoria y } \\
\text { atención, presentándose mayores dificultades } \\
\text { en CCc. }\end{array}$ \\
\hline $\begin{array}{l}\text { Salamanca- } \\
\text { Zarzuela et al., } \\
2018\end{array}$ & España & $\begin{array}{l}64 \mathrm{~N} \text { total }(38 / 26) \\
28 \text { CCC, } 36 \mathrm{CCA} \\
\text { Edad:2-18 }\end{array}$ & TD II & $\begin{array}{l}\text { El área del lenguaje se encontraba alterada en } \\
15,6 \% \text {, el motor en } 10,9 \% \text { y trastornos de la } \\
\text { interacción social en } 8 \% \text { de los pacientes. } \\
\text { Existen diferencias significativas entre CCC y } \\
\text { CCA. }\end{array}$ \\
\hline
\end{tabular}

Fuente: elaboración propia a partir de los estudios seleccionados sobre el neurodesarrollo de menores y jóvenes con cardiopatías.

BSID-II: The Bayley Scales of Infant Development 2nd edition; MDI: Mental Development Index; PDMS: Peabody Developmental Motor Scales; ASQ: Ages \& Stages Questionnaire; WISC-IV: Wechsler Intelligence Scale for Children, 4th edition; ROCFT: the Rey-Osterrieth Complex Figure Test; ZNA: the Zurich Neuromotor Assessment; HRQoL: Health-related quality of life; BSID III: Scales of infant and Toddler Development, 3rd edition; ITSEA: The Infant Toddler Social and Emotional Assessment; PDI: Psychomotor Developmental Index; BRIEF: Behavior Rating Inventory of Executive Function; CBCL: Child Behavior Checklist; Peds-QL: Pediatric Quality of Life Inventory; QOL: Quality of life; M-ABC-2: Movement Assessment Battery for Children, second edition;WPPSI-R: the Revised Wechsler Preschool and Primary Scale of Intelligence; CBCL: the Child Behavior Checklist; NePsy: Developmental Neuropsychological Assessment, second edition; Neuropsi: Neuropsychological Test for Memory and Attention; TD II: Test de Denver; WAIS: Wechsler Adult Intelligence Scale-Fourth Edition; ASR: Adult Self Report; YSR: Youth Self Report (YSR).

MF: Motricidad Fina; MG: Motricidad Gruesa; CC: Cardiopatías Congénitas; GC: Grupo Control sano; CCC: Cardiopatías Congénitas Cianóticas; CCA: Cardiopatías Congénitas Acianóticas; Cl: Cociente Intelectual; CEC: Cirugía por Circulación Extracorpórea; CIC:C Cardiopatía Isquémica Compleja; VU: Ventrículo único; CCs: Cardiopatía Congénita Simple; CCc: Cardiopatía Congénita Compleja; SNC: Sistema Nervioso Central; UVH: Defecto Cardiaco Univentricular; HN: Hemodinámicamente normales; HI: deficientes hemodinámicamente. 


\section{Resultados}

La literatura científica de la última década, centrada en el desarrollo de las personas con cardiopatías congénitas, revela información sobre las morbilidades y comorbilidades presentes en las mismas.

Los resultados obtenidos tras la revisión sistemática se reportan en base a dos subgrupos de edad, de 3 a 84 meses y de 85 a 192 meses.

Es importante mencionar que la mayoría de las investigaciones del primer subgrupo utilizan la Escala de Desarrollo Infantil Bayley-II y III, entre otras, como método de evaluación del neurodesarrollo de su muestra. Esta escala valora las áreas evolutivas más importantes: cognitiva, lenguaje y motora. En los estudios del segundo subgrupo predomina la utilización de la Escala de Inteligencia de Wechsler para niños, $3^{\mathrm{a}}$ y $4^{\mathrm{a}}$ edición (WISC-III y IV), entre otras. Esta se centra en la evaluación de la aptitud intelectual de niños y adolescentes.

En el área motora, el primer grupo presenta un desarrollo inferior (Sananes et al., 2012; Hallioglu et al., 2015; Latal et al., 2016; Rollins y Newburguer, 2017; Salamanca-Zarzuela et al., 2018) aunque la motricidad gruesa tiende a normalizarse con el propio desarrollo evolutivo. Se hacen evidentes mayores dificultades en la motricidad fina cuando el sujeto crece, obteniendo menores puntuaciones en el desempeño escolar (Gerstle et al., 2016) aunque también tienen tendencia a normalizarse (Sterken et al., 2016).

Investigaciones recientes muestran como los infantes con CC presentan lentificaciones en el área comunicativa-lingüística, especialmente en el subárea expresiva (Hallioglu et al., 2016) en comparación con el GC, y tienen mayor riesgo de déficits en el habla (Sananes et al., 2012).

En el área cognitiva, la literatura científica refuta que el grupo con CC alcanza puntuaciones inferiores en el $\mathrm{Cl}$ en comparación con el GC (Sterken et al., 2016). En otro estudio realizado por Hallioglu et al. (2015) se muestra como los pacientes con CC presentaron un desarrollo normal-lento en el área cognitiva en comparación con el grupo de control, situándose estos últimos dentro del promedio poblacional.

Existen contradicciones en los resultados alcanzados en distintas investigaciones. Algunos autores señalan que no existen diferencias significativas en los dominios del desarrollo entre los menores con CC y el GC (Chen et al., 2015) mientras otros indican que los menores con CC obtienen puntuaciones inferiores en todas las áreas del desarrollo en comparación con el GC (Hallioglu et al., 2015; Goldsworthy et al., 2016; Rollins y Newburguer, 2017). El estudio realizado por Sterken et al. (2016) demuestra que no existe un aumento en los déficits neurocognitivos entre los 4 y los 7 años de edad en comparación con los controles sanos.

Se evidencian diferencias significativas entre los menores que presentan una cardiopatía acianótica y cianótica, obteniéndose peores resultados en esta última en el test de Denver II (Salamanca-Zarzuela et al., 2018).

Las medidas biométricas como la talla, el peso, el perímetro cefálico (Sterken et al., 2016), la circunferencia media del brazo y el pliegue cutáneo del tríceps de los menores con CC pueden ser inferiores (Hallioglu et al., 2015) y no tienen porqué repercutir en la función neurocognitiva (Sterken et al., 2016). Sin embargo, algunos estudios hacen evidente la relación entre los criterios biomédicos y los resultados alcanzados en el desarrollo 
del menor con $\mathrm{CC}$, asociándose menor puntuación antropométrica en perímetro cefálico con puntuaciones inferiores en MDI y una menor talla con un peor PDI (Medoff-Cooper et al., 2016).

La actividad convulsiva se vinculó a un desarrollo motor inferior (Latal et al., 2016) y la necesidad de varias intervenciones quirúrgicas, durante los primeros años de vida, se asoció a mayores alteraciones en el neurodesarrollo (Goldsworthy et al., 2016). El estudio realizado por Hallioglu et al. (2015) señala que no existen diferencias significativas entre los pacientes con CC que fueron intervenidos antes o después de los 12 meses de edad, aunque los infantes sometidos a cirugía cardiaca tienen un desarrollo deteriorado dos años después de la misma (Sananes et al., 2012).

Otros estudios exponen la relación existente entre factores familiares y la evolución del desarrollo del menor. Los padres reportan que sus hijos presentan lentificaciones en el lenguaje expresivo y receptivo (frases, gestos, comprensión de vocabulario) (Rollins y Newburguer, 2017) y que esto se ve influenciado por el nivel de educación materna (Goldsworthy et al., 2016) ya que es la cuidadora principal del menor.

La revisión sistemática del segundo grupo, cuyo rango de edad es comprendido entre 85 y 192 meses, recoge que estas personas alcanzan menores puntuaciones en las funciones ejecutivas, en el desempeño escolar, razonamiento perceptivo, integración visomotora, memoria de trabajo y en las habilidades motoras (Schaefer et al., 2013; Sarrechia et al., 2015; Gerstle et al., 2016).

La competencia motora muestra que presentan dificultades para imitar las posiciones de las manos, memorizar y ejecutar secuencias motoras (Sarrechia et al., 2015) y en la integración viso-motora pero no en el equilibrio estático (Schaefer et al., 2013). Esto se acompaña de dificultades en la comprensión de instrucciones, en la repetición de pseudopalabras y en la generación de las mismas (Sarrechia et al., 2015).

Los infantes y los adolescentes con CC presentan un alto riesgo de desarrollar alteraciones en la memoria, en las funciones ejecutivas y problemas de atención (González-González et al., 2018), presentando dificultades en el aprendizaje, requiriendo programas educativos individualizados y mostrando una competencia escolar inferior (Gerstle et al., 2016). Los padres reportan que sus hijos e hijas presentan dificultades en la lecto-escritura y en la materia instrumental de matemáticas, requiriendo tutorías adicionales y apoyos escolares (Schaefer et al., 2013).

Autores como Schaefer et al. (2013) señalan que existen rangos normales del Cl pero el estudio realizado por Murphy et al. (2015) indica que los menores con CC tienen un $\mathrm{Cl}$ inferior al grupo de control, presentando más problemas atencionales y dificultades de aprendizaje (Schaefer et al., 2013).

Por último, mencionar que las personas con CC pueden presentar problemas socioemocionales. Estudios recientes demuestran que a los 24 meses de edad se alcanzaron puntuaciones normales con riesgo de internalización y externalización y a los 108 meses, el grupo con defecto cardiaco univentricular mostró mayores problemas de externalización (Sarrechia et al., 2015; Goldsworthy et al., 2016). 


\section{Discusión}

En la última década se han producido avances quirúrgicos que han reducido las tasas de mortalidad de los menores con CC (Marino et al., 2012). Tras esto, se comienzan a evidenciar alteraciones en las distintas áreas del desarrollo.

Distintos estudios coinciden en que los menores con cardiopatías presentan un desarrollo motor inferior en comparación con el grupo de control (Schaefer et al., 2013; Chen et al., 2015; Rollins y Newburger, 2017), independientemente de la edad que tengan. Según Sananes et al. (2012) el desarrollo motor grueso progresa durante el crecimiento del menor, reduciéndose el fino, pero otros autores recogen que se alcanzan puntuaciones inferiores en esta área según aumenta la edad de los participantes (Goldsworthy et al., 2016; Medoff-Cooper et al., 2016; Latal et al., 2016).

La disminución progresiva en la motricidad fina es preocupante porque esta habilidad es muy importante para el éxito académico en los primeros años escolares, cuando los niños comienzan con el aprendizaje de números y letras, entre otras cosas (Sananes et al., 2012).

Otros estudios recogen que los menores con CC obtienen puntuaciones inferiores en las funciones ejecutivas, en la planificación, en las habilidades perceptivas-visuales y posteriormente en las académicas (Murphy et al., 2015; Gerstle et al., 2016; González-González et al., 2018) ya que se encuentran alteraciones en la atención sostenida y en la memorización (Miatton et al., 2007). Sin embargo, Sterken et al. (2016) indican que las funciones ejecutivas y la flexibilidad cognitiva se normalizan cuando van creciendo los sujetos.

También existen contradicciones al analizar el $\mathrm{Cl}$ de los menores y adolescentes con CC pues algunos autores sugieren que está situado en un desarrollo normal-bajo (Majnemer et al., 2006; Miatton et al., 2007; Gerstle et al., 2016; Rollins y Newburguer, 2017) y otros que se encontraba dentro del promedio (Brosig et al., 2007; Calderon et al., 2012; Sterken et al., 2016). Esto indica que es necesario continuar con nuevas vías de investigación que permitan generalizar los resultados encontrados.

Los menores con CC están en riesgo de presentar morbilidades en el área del lenguaje (Bellinger y Newburguer, 2010; Salamanca-Zarzuela et al., 2018) y esto puede repercutir en sus logros educativos. Estos retrasos pueden deberse al estado físico deficiente del niño y asociarse con las restricciones existentes en la exploración del entorno (Monfort y Juárez, 2010; Sananes et al., 2012).

Los niños con CC, después de la cirugía, se encuentran en alto riesgo de retraso del desarrollo pues algunos necesitan intervenciones quirúrgicas en edades tempranas (Hövels-Gürich et al., 2007; Goldsworthy et al., 2016). En estudios anteriores se evidenció que la duración de la cirugía por circulación extracorpórea (CEC) predecía el nivel de autonomía ya que cuanto más se prolongue en el tiempo la intervención, mayores serán las alteraciones en el desarrollo (Brosig et al., 2007) y se demostró que el momento de la intervención repercute en el desarrollo del menor (Majnemer et al., 2006). El peso, la talla y el perímetro cefálico parecen ser inferiores en los menores con CC que en los grupos de control (Miatton et al., 2007; Medoff-Cooper et al., 2016), pudiendo ser un indicativo más para predecir el neurodesarrollo de los sujetos con CC. 
Los padres siempre son un estimulo en el desarrollo de sus hijos, presenten o no CC. Sin embargo, se debe resaltar que estos están sometidos a altos niveles de estrés por la enfermedad de su hijo, lo cual puede generar sobreprotección en el menor y mayor preocupación sobre la evolución de este, mostrando un mayor impacto negativo en la familia (Brosig et al., 2007). Sin embargo, otro estudio (Rollins y Newburger, 2017) demuestra que los padres reportan lentificaciones en el desarrollo del lenguaje expresivo y receptivo. Esto resalta la necesidad de investigar sobre esta temática y demostrar si existe sobreprotección en el hogar, conocer los estilos de crianza, los niveles de estrés de los cuidadores principales y si la información que reportan es verídica o está condicionada.

Todo esto puede producir dificultades en las habilidades académicas, especialmente en la escritura, la lectura, ortografía y las matemáticas (Miatton et al., 2007; Schaefer et al., 2013) aunque asistan a la escuela con regularidad. Los padres de los menores con CC reportan retrasos en la comprensión del vocabulario y señalan que tienen una competencia escolar inferior (Gerstle et al., 2016).

En relación con la regulación emocional, se observa que los niños con CC tienen problemas de internalización y externalización (Brosig et al., 2007; Sarrechia et al., 2015; Gerstle et al., 2016). Por tanto, queda claro que los menores que presentan $\mathrm{CC}$ tienen dificultades en el entorno académico, en la regulación conductual y en las distintas áreas del desarrollo, tanto en la primera infancia como en edades posteriores.

\section{Conclusiones}

Es necesario continuar investigando sobre esta temática para poder generalizar resultados y demostrar si existen o no dificultades del aprendizaje en los discentes con cardiopatías congénitas, sin síndromes asociados ni síndromes genéticos, que pudieron generarse en edades tempranas. Para ello, es preciso seguir analizando el neurodesarrollo de los menores con CC y descubrir si se presentan o existe el riesgo de que presenten lentificaciones en las distintas áreas para poder dotarles de intervenciones tempranas que permitan mejorar su desarrollo.

La limitación principal de esta revisión sistemática es la escasez de estudios incorporados, pues son los que cumplían con los criterios de inclusión. Se han encontrado numerosas investigaciones que estaban formadas por muestra prematura (< 36 semanas de edad gestacional), con síndromes genéticos y discapacidades, que han sido descartadas pues se pretende demostrar las lentificaciones que provoca la propia cardiopatía congénita.

Como propuesta de mejora, es preciso realizar una revisión sistemática de menores que presenten anomalías cardiacas y cuyo nacimiento se produzca a partir de la semana 37 de edad gestacional.

Por último, es necesario que se realicen distintas investigaciones, transversales y longitudinales, en las que se utilicen las mismas escalas de valoración, en distintos rangos de edad, a fin de obtener resultados equiparables entre la muestra poblacional y, por ende, conocer las características del neurodesarrollo de los menores con CC para atender a las necesidades que puedan presentar. 


\section{Referencias bibliográficas}

American Heart Association (2018): Understand Your Risk for Congenital Heart Defects. Retrieved (en línea). $<$ https://www.heart.org/en/health-topics/congenital-heart-defects/understand-your-risk-for-congenital-heartdefects $>$, acceso 12 de febrero de 2019.

Bellinger, D. y Newburguer, J. (2010): "Neuropsychological, psychosocial, and quality-of-life outcomes in children and adolescents with congenital heart disease". Progress in Pediatric Cardiology, 29 (2): 87-92.

Brosig, C. et al. (2007): "Neurodevelopmental outcome in preschool survivors of complex congenital heart disease: implications for clinical practice". Journal of Pediatric Health Care, 21 (1): 3-12.

Calderon, J. et al. (2012): "Impact of prenatal diagnosis on neurocognitive outcomes in children with Transposition of the Great Arteries". Journal of Pediatric, 161 (1): 94-98.

Cardiopediamarañón (2017): Área del corazón infantil HGUGM (en línea). <https://www.cardiopedhgugm.com/ cardiopat\%C3\%ADas-congénitas/cia/>, acceso 20 de octubre de 2019.

Chen, C. et al. (2015): "Infants with complex congenital heart diseases show por short-term memory in the mobile paradigm at 3 months of age". Infant Behavior and Development, 40: 12-19.

Ferencz, C. y Neill, C. A. (1992): "Cardiovascular malformations: prevalence at birth. In: Freedom RM, Benson L, Smallhorn J, eds". Neonatal heart disease, 1: 19-29.

García, R. et al. (2018): "Acute Kidney injuri following first stage pallation in hypoplastic left heart syndrome: hybrid versus Norwood palliation”. Cardiology in the Young, 28 (2): 261-268.

Gaynor, W. et al. (2007): "Patient characteristics are important determinants of neurodevelopmental outcome at one year of age after neonatal and infant cardiac surgery". Surgery for Congenital Heart Disease, 133 (5): 13441353.

Gerstle, et al. (2016): "Executive Functioning and School Performance among Pediatric Survivors of Complex Congenital Heart Disease". The Journal of Pediatrics, 173: 154-159.

Goldsworthy, M. et al. (2016): "Relationship between social-emotional and neurodevelopment of 2-year-old children with congenital heart disease". Congenit Heart Dis, 11 (5): 378-385.

González-González, G. et al. (2018): "Assessment of cognitive performance among Mexican children and adolescents afflicted by simple to complex congenital heart diseases. Preliminary study". Progress in Pediatric Cardiology, 48: 93-97.

Greco, R. (2016): Jornada de Cardiopatías Congénitas. Situación actual de la cirugía cardiaca de las principales cardiopatías (en línea). <http://original.livestream.com/grupocto/video?clipld=pla_cded1b26-a689-470bbdd4-29f1f9024a3a\&utm_source=Islibrary\&utm_medium=ui-thumb>, acceso 9 de febrero de 2017.

Hallioglu, O. et al. (2015): "Evaluation of neurodevelioment using Bayley-III in children with cyanotic or hemodynamically impaired congenital heart disease". Congenit Heart Dis, 6 (10): 537-541.

Harvey, K. et al. (2013): "Experiences of mothers of infants with congenital heart disease before during and after complex cardiac surgery". Heart Lung, 42 (6): 399-406.

Hövels-Gürich, H. et al. (2007): "Long-term outcome of speech and language in children after corrective surgery for cyanotic or acyanotic cardiac defects in infancy". European Journal of Paediatric Neurology, 12 (5): 378-386. 
Latal, B. et al. (2016): "Postoperative amplitude-integrated electroencephalography predicts four-year neurodevelopmental outcome in children with complex congenital heart disease”. The Journal of Pediatrics, 178: 55-60.

Majnemer, A. et al. (2006): "Long-Term neuromotor outcome at school entry of infants with congenital heart defects requering open-heart surgery". J Pediatr, 148 (1): 72-77.

Marino, B. et al. (2012): "Neurodevelopmental outcomes in children with congenital heart disease: evaluation and management. A scientific statement from the American Heart Association”. Circulation, 126 (9): 1143-1172.

Martínez-Biarge, M. et al. (2013): "Neurodevelopmental outcome in children with congenital heart disease". Seminars in Fetal \& Neonatal Medicine, 5 (18): 279-285.

Medoff-Cooper, B. et al. (2016): "The Association among feeding mode, growth, and developmental outcomes in infants with complex congenital heart disease at 6 and 12 months of age". The Journal of Pediatrics, 169: 154-159.

Miatton, M. et al. (2007): "Neuropsychological performance in school-Aged children with surgically corrected congenital heart disease". The Journal of Pediatrics, 1 (151): 73-78.

Monfort, M. y Juárez, A. (2010): El niño que habla. El lenguaje oral en preescolar. Madrid: CEPE.

Murphy, L. et al. (2017): "Cognitive and attentional functioning in adolescents and young adults with Tetratology of Fallot and d-transposition of the great arteries". Child Neuropsychology, 23 (1): 99-110.

Pasquali, S. K. et al. (2011): "Center variation in hospital costs for patients undergoing congenital heart surgery". Circ Cardiovasc Qual Outcomes, 4 (3): 306-312.

Polat, S. et al. (2011): "Evaluation of growth and neurodevelopment in children with congenital heart disease". Pedriatrics, 53 (3): 345-349.

Rollins, C. y Newburger, J. (2017): “Neurodevelopmental outcomes in congenital heart disease”. Cardiology Patient Page, 130 (14): 124-126.

Robbins, J. M. et al. (2007): "Hospital stays, hospital charges, and in-hospital deaths among infants with selected birth defects-united states". MMWR, 56 (2): 25-29.

Salamanca-Zarzuela, B. et al. (2018): "Desarrollo psicomotor en pacientes con cardiopatía congenital grave". Revista de Neurología, 66 (12): 409-414.

Sananes, R. et al. (2012): "Neurodevelopmental outcomes after open heart operations before 3 months of age". Pediatric Cardiac, 93 (5): 1577-1583.

Sarrechia, I. et al. (2015): "Neurodevelopmental outcome after surgery for acyanotic congenital heart disease". Research in Developmental Disabilities, (45-46): 58-68.

Schaefer, C. et al. (2013): "Neurodevelopmental outcome, psychological adjustment and quality of life in adolescents with congenital heart disease”. Developmental Medicine \& Child Neurology, 12 (55): 1143-1149.

Sterken, C. et al. (2016): “Neurocognitive development after pediatric heart surgery”. Pediatrics, 137 (6): e20154675.

Williams, J. et al. (2015): "Neurodevelopmental outcomes after cardiac surgery in infancy". Pediatrics, 135 (5): 817-825. 